\title{
Clinical features and neurobiological aetiology of obsessive compulsive symptoms in patients with Parkinson's disease
}

\begin{abstract}
Obsessive-compulsive symptoms and obsessive-compulsive disorder have often been observed in patients with Parkinson's disease. Although dysfunction of basal ganglia has a key role in creating obsessive compulsive behavior in patients with Parkinson's disease, many researchers argue that separate and parallel frontostriatal circuits are likely to cause the clinical symptoms of Parkinson's disease and obsessive compulsive disorder. As the degree of severity of illness increases, obsessive compulsive traits most notably change of checking, doubting and cleaning subscales - become more and more evident in patients with Parkinson's disease. It has been suggested that obsessive compulsive symptoms which have been observed in the progression of the illness are related to neurochemical changes taking place at the basal ganglia phase rather than nigrostriatal dopaminergic deficiency. In the present review clinical features and neurobiological aetiology of obsessive compulsive symptoms in patients with Parkinson's disease have been discussed on the basis of scientific evidence published in recent literature. The research papers and case reports in the Pub med database have been searched without date restriction.
\end{abstract}

Keywords: Parkinson's disease, obsessive compulsive disorder, aetiology, basal ganglia
Volume 8 Issue 6 - 2018

\author{
Sefa Vayısoğlu \\ Adana Dr. Ekrem Tok Mental Health and Diseases Hospital, \\ Turkey
}

Correspondence: Sefa Vayısoğlu, Adana Dr. Ekrem Tok Mental Health and Diseases Hospital,Turgut Ozal Bulvari no 230/I Adana,Turkey, Email sefavayisoglu@gmail.com

Received: July 26, 2018 | Published: November 29, 2018
Abbreviations: PD, Parkinson disease; NMS, non-motor symptoms; OCD, obsessive-compulsive disorder; ICD, impulse control disorders; TS, tourette's syndrome

\section{Introduction}

Most patients with Parkinson disease (PD) have non-motor symptoms (NMS) which include the ones of psychiatric disorders and NMS dominate the prodromal phase of PD and some may serve as clinical biomarkers of PD. NMS can be dopaminergic, nondopaminergic, of genetic origin or drug induced. The syndromic nature of PD is underpinned by non-motor subtypes which are likely to be related to specific dysfunction of cholinergic, noradrenergic, serotonergic pathways in the brain, not just the dopaminergic pathways. ${ }^{1}$ One or more psychiatric disorders arise in approximately $90 \%$ of patients with Parkinson's disease throughout the course of the disease. The clinical symptoms of anxiety and depression can be observed in the early stages of motor symptoms in $30 \%$ of the patients. Psychosis affects $25 \%$ of patients. ${ }^{2}$ Obsessive-Compulsive disorder (OCD) is less common compared to the other psychiatric illnesses; however, it can be seen among Parkinson's patients. Parkinson's disease is characterized by fronto-basal ganglia circuit dysfunction. ${ }^{3}$ A similar dysfunction also plays a role in the pathophysiology of obsessive-compulsive disorder (OCD). ${ }^{4,5}$ Some studies indicate that this common dysfunction may be the reason why OCD is very common among patients with Parkinson's disease. ${ }^{6-9}$ The purpose of this review is to emphasize the clinical features and biological etiology of the OCD symptoms observed in patients with Parkinson's disease to raise awareness regarding the subject. For this purpose, the authors used the Pub Med search engine by searching the keywords 'Parkinson's Disease, obsessive-compulsive disorder, basal ganglia'.
The inclusion criteria were: being published in English and containing the aforementioned keywords in the title/abstract section. The accessed published material, and the articles and case reports cited by these materials were reviewed.

\section{Clinical aspects}

A multicenter case-control designed study which is aiming to assess factors associated with impulse control disorders (ICD) in PD reported that PD patients with ICD showed greater obsessivecompulsive symptoms when compared with those patients without ICD.$^{10}$ In a study aiming to compare obsessive-compulsive complaints in patients with OCD, Tourette's Syndrome (TS) and Parkinson's disease using the Maudsley Obsessive-Compulsive Inventory (MOCI) and the Short Form of the Hamburg Obsessive-Compulsive Inventory (HZI-K), Parkinson's patients were compared to controls in terms of MOCI subscales. There was no difference between the groups. When compared with OCD patients, it was found that the scores of Parkinson's were significantly lower. Also, the scores of Parkinson's patients were lower than the TS patients in all sub-scales except for 'washing, cleaning' and 'slowness'. When the Parkinson's patients were compared to the controls through HZI-K, the score for the tidying subscale was higher for the patient group. In addition, when Parkinson's patients were compared with OCD and TS patients, it was found that their total HZI-K scores were significantly lower. ${ }^{11}$ In a study, the severity of obsessive-compulsive complaints was associated with motor disability in Parkinson's disease. ${ }^{9}$ In another study, the on-off phenomenon observed in Parkinson's disease was found to be associated with complex manners and organized rituals. ${ }^{7}$

In a study in which 72 non-demented idiopathic Parkinson's patients with Parkinson's disease were investigated using MOCI (Maudsley 
Obsessive-Compulsion Inventory) and LOI (Leyton Obsessional Inventory); the patients were compared with healthy controls matched for age, gender, and educational level. It was found that the patients with mild Parkinson's symptoms did not have obsessive-compulsive complaints, while patients with a severe disease did. From this determination, it has been concluded that; as Parkinson's disease progresses clinically, obsessive-compulsive complaints increase, which may be linked to the neurochemical changes that occur in the basal ganglia circulation as the disease progresses. ${ }^{12}$

Parkinson's patients $(n=100)$ were compared to healthy controls $(n=100)$ in terms of personality disorders, and it was determined that the most common personality disorder seen among Parkinson's patients was obsessive-compulsive personality disorder (OCPD). This condition was found to be independent of the duration of the disease and the dopaminergic treatment. ${ }^{13}$

A study compared newly diagnosed Parkinson's patients who had not received anti-Parkinson medication before $(n=40)$ and the ageand gender-matched control group $(n=40)$ to determine the frequency of OCPD. It was determined that OCPD was the most common personality disorder among the patients, $55 \%$ patients having OCPD. As these results were found among newly diagnosed patients that hadn't received anti-Parkinson treatment, the OCPD is said to be an early finding of Parkinson's disease that is independent of drug treatment. ${ }^{14}$

A study evaluated the obsessive-compulsive complaints and OCD among 69 idiopathic Parkinson's patients without dementia and a paired control group using the Yale-Brown Obsession Compulsion Scale (Y-BOCS) and structured clinical interviews. The two groups were not found to be different regarding obsessive-compulsive complaints. Also, the severity of Parkinson's disease was not found to be associated with obsessive-compulsive symptoms. Based on these findings, it was suggested that the fronto-striatal circuits that take part in the pathophysiology of Parkinson's disease and OCD may be different. ${ }^{15}$

In a study; OCD, obsessive-compulsive complaints, and related symptoms (tic disorder, trichotillomania, body dysmorphic disorder) of Parkinson's patients were compared with a healthy control group. The two groups were not found to be different; however, the leftsided motor symptoms were found to be associated with obsessivecompulsive complaints (such as symmetry and ordering/tidying). This finding led to the hypothesis that the right hemisphere had a more prominent role in the formation of obsessive-compulsive complaints. ${ }^{16}$

A study evaluated procedural learning among OCD patients by comparing Parkinson's and Major Depressive Disorder patients. It was concluded that there is a functional disorder in the striatal system among OCD patients, and that the inappropriate open processing that is observed in OCD patients led to the striatal system dysfunctions. ${ }^{17}$

A study investigated the association of the impulse control behaviors (ICB) that are continuous, that decrease or emerge before and after subthalamic deep brain stimulation with clinical and demographic factors. It was found that the symptoms of the patients with OCPD characteristics were within the continuous ICB category. ${ }^{18}$

Punding is a term that was coined originally to describe complex prolonged, purposeless, and stereotyped behavior in chronic amphetamine users. A structured interview of 50 patients with higher dopamine replacement therapy requirements from 123 unselected patients with PD from a PD clinic identified 17 (\%14) patients with punding. Punding was acknowledged as disruptive and unproductive by the patients themselves, but forcible attempts by family to interrupt the behavior led to irritability and dysphoria. It is commonly associated with the syndrome of dopamine dysregulation and is phenomenologically distinct from obsessive-compulsive disorder. 19

A study which was to evaluate the frequency of personality disorders in PD patients and in a group of healthy control found that PD patients presented a high frequency of obsessive-compulsive personality disorder that does not seem to be related with both disease duration and dopaminergic therapy. ${ }^{20}$

\section{Biological etiology}

Lethargic encephalitis, defined by von Economo in 1917, is a disease that is characterized by Parkinsonian and obsessivecompulsive symptoms. The histopathological findings include lesions in the basal ganglia. These findings have led to the conclusion that OCD may be more frequently observed in people with Parkinson's disease. $^{21}$

Several structural and functional neuroimaging studies have found that of obsessive-compulsive disorder is associated with basal ganglia dysfunction. ${ }^{22,23}$ The lesions in the basal ganglia lesions lead to the formation of obsessive-compulsive complaints that are similar to OCD symptoms. ${ }^{24}$ It is a well-known fact that several fronto-basal dysfunction symptoms are observed in patients with Parkinson's disease. Limbic circuit dysfunction in Parkinson's patients may also be responsible from the accentuation of obsessive-compulsive findings.

A 51-year-old female patient was followed with OCD for 33years and with PD for 5years. She had severe motor complications induced by levodopa despite adequate treatment for 4 years. After the placement of a stimulating electrode to the anteromedial part of the subthalamic nucleus (STN), the obsessive-compulsive complaints disappeared after two weeks and the Parkinsonian symptoms slightly improved. A 50-year-old male patient that was followed with OCD for 40years and with PD for 16years underwent a similar procedure. The electrode that was placed in the anteromedial part of STN completely resolved the OCD symptoms and the obsessions of tidying and controlling majorly reversed. The findings related to these two patients suggest that the high-frequency stimulation of the subcortical limbic circuit can improve the functionality of OCD patients. ${ }^{25}$

The commonly accepted view is that different and parallel cortico-striato-thalamic cycles corresponding to the cortex, striatum and thalamus topographies have different functions. ${ }^{26,27}$ Striatum is not a functionally homogeneous structure. The different parts of the striatum are in communication with different regions of the cortex and the thalamus. The neuroimaging studies have found that the hyperactivity of the orbit frontal cortex and the caudate nucleus is linked to obsessions. The main pathology in Parkinson's disease concerns the pars compacta of substantia nigra. The melatonincontaining neurons of the substantia nigra produce dopamine and project to the striatum. When compared with the putamen, caudate, it is significantly dopamine poor, thus, it is not affected as much from the loss of dopaminergic cells. Functional imaging studies provide varying results regarding Parkinson's disease. ${ }^{3}$ Some indicate that the healthy controls aren't different from the patient group; ${ }^{28}$ and some find that the patients have hypermetabolism in the lenticular nucleus 
and the thalamus, and hypometabolism in the premotor and parietooccipital regions. ${ }^{29}$ Some other studies have found basal ganglia perfusion that improved with levodopa treatment. ${ }^{30}$ A 59-year-old Parkinson's patient with treatment-resistant OCD (TR-OCD) was treated with deep brain stimulation (DBS) to the ventral capsule (VC) and the ventral striatum (VS). The symptoms of the patient improved after DBS. ${ }^{31}$

Studies with genetically modified mice with corticostriatal dysfunction and OCD-like behavior have found that the most important factor that leads to the development of OCD-like symptoms was a common dysfunction in glutaminergic signalization, including striatum dysfunction. These mouse models include the transgenic D1CT-7 model (the hyperactivation of the glutaminergic input that is produced by the chronic potentialization of the dopamine type 1 receptor-expressing neurons to the striatum), the Sapap3/Dlgap3 deletion model (disrupted corticostriatal glutamatergic transmission, etc) and Slitrk5 deletions model (increased orbitofrontal cortex activity, decreased striatal volume, and disrupted corticostriatal transmission)..$^{32-36}$

OCD is mainly observed in neurodegenerative disorders, such as Huntington's disease ${ }^{37}$ and Tourette's syndrome. ${ }^{38}$ The motor symptoms in Parkinson's disease are the complete opposite of the motor hyperactivation symptoms observed in Tourette's syndrome and Huntington's disease. However, levodopa, which is used in the treatment of Parkinson's, may induce dyskinesia. A Parkinson's patient with advanced obsessive-compulsive symptoms and advanced dyskinesia was observed three months after a pallidotomy operation. The results suggested that similar neurobiological mechanisms underlay both the obsessive-compulsive symptoms and the dyskinesia. ${ }^{39}$ Nevertheless, there are studies that indicate that the obsessive-compulsive symptoms observed in Parkinson's patients can be seen as an OCPD component that is independent of antiParkinsonian treatment. ${ }^{13,14}$

\section{Discussion}

OCD symptoms are reflections of the pathological and repeated behavioral programs that root from basal ganglia dysfunction. ${ }^{40,41}$ After the development of a unilateral limited vascular lesion in the left putamen of an idiopathic Parkinson's patient, the patient developed obsessions in a few days, and compulsions in a few weeks. The severity of these symptoms increased with time. As illustrated by this case, the data regarding lesions in the central nervous system of Parkinson's patients indicate a potential association between OCD symptoms and basal ganglia. ${ }^{42}$

Punding induced by large quantities of DRT in PD provides a clinical syndrome directly relevant for testing the link between OCD and dorsal striatal hyperdopaminergia in humans. The study conducted by Evans et al. ${ }^{22}$ suggests that there are clear similarities, but also important differences between punding and OCD. Punding, like certain ritualistic behaviors seen in OCD, is purposeless, representing dissociation between knowledge and performance. In addition, performance of complex stereotypies produces feelings of calm and relief, somewhat similar to OCD. In contrast with OCD, punding is not associated with obsessionality, including religious, aggressive, sexual, checking, symmetry, ordering, counting, contamination and cleaning/ washing compulsions. ${ }^{43}$ OCD symptoms related to basal ganglia dysfunction and OC-like symptoms in punders (especially hoarding) represent stereotyped behaviours resulting from the potentiation of psychomotor processes and habitual, routine behaviours. ${ }^{21}$

In a Parkinson's patient, the DBS treatment applied to the reward circuit VC/VS has corrected treatment-resistant obsessive-compulsive symptoms. ${ }^{31}$ This suggests that, together with STN, this region may also take part in the path physiology of the obsessive-compulsive symptoms observed in Parkinson's patients.

Other than psychiatric disturbances, such as depression, anxiety, psychosis and impulse control disorder; obsessive-compulsive symptoms can also be observed among Parkinson's patients. Based on the reviewed studies in this article, it can be suggested that the neurobiological dysfunction in OCD is closely related to the frontostriatal dysfunction in Parkinson's disease, and that this association leads to the common clinical symptoms. Further clinical and imaging studies on the neural circuit disorder will contribute to a better understanding of the physiopathology of obsessive-compulsive symptoms, and to the development of effective psychotropic drug therapies on the basis of this knowledge to increase the quality of life of the patients with Parkinson`s disease.

\section{Acknowledgments}

None.

\section{Conflicts of interest}

The author declares no conflicts of interest.

\section{References}

1. Titova N, Chaudhuri KR. Non-motor Parkinson disease: new concepts and personalized management. Med J Aust. 2018;208(9):404-409.

2. Sadock BJ, Sadock VA. Comprehensive Textbook of Psychiatry (Çev. ed: H. Aydın, A. Bozkurt) Güneş Kitabevis. 2007. 408 p.

3. Rauch SL, Savage CR. Neuroimaging and neuropsychology of the striatum. Psychiatr Clin N Am. 1997;20:741-768.

4. Rauch SL, Whalen PJ, Dougherty D, et al. Neurobiologic models of obsessive-compulsive disorder. Obsessive-Compulsive Disorders: Practical Management. Mosby: St Louis; 1998. p. 222-253.

5. Saxena S, Brody AL, Schwartz JM, et al. Neuroimaging and frontosubcortical circuitry in obsessive-compulsive disorder. Br J Psychiatry Suppl. 1008;35:26-38.

6. Alegret $\mathrm{M}$, Junque $\mathrm{C}$, Valldeoriola $\mathrm{F}$, et al. Obsessive-compulsive symptoms in Parkinson's disease. J Neurol Neurosurg Psychiatry. 2001;1:58-60

7. Hardie RJ, Lees AJ, Stern GM. On-off fluctuations in Parkinson's disease. Brain. 1984;107(2):487-506.

8. Lees AJ. The neurobehavioral abnormalities in Parkinson's disease and their relationship to psychomotor retardation and obsessional compulsive disorders. Behav Neurol. 1989;2(1):1-11.

9. Tomer R, Levin BE, Weiner WJ. Obsessive-compulsive symptoms and motor disturbances in Parkinson's disease. Neuropsychiatry Neuropsychol Behav Neurol. 1993;6:26-30.

10. Voon V, Sohr M, Lang AE, et al. Impulse Control Disorders in Parkinson Disease: A Multicenter Case-Control Study. Ann Neurol. 2011;69(6):986-996.

11. Muller N, Putz A, Kathmann N, et al. Characteristics of obsessivecompulsive symptoms in Tourette's syndrome, obsessive-compulsive disorder and Parkinson's disease. Psychiatr Res. 1997;70(2):105-114. 
12. Alegret $M$, Vendrell $P$, Junque $C$, et al. Effects of unilateral posteroventral pallidotomy on 'on-off' cognitive fluctuations in Parkinson's disease. Neuropsychologia. 2000;38(5):628-633.

13. Nicoletti A, Luca A, Raciti L, et al. Obsessive compulsive personality disorder and Parkinson's Disease. PLoS ONE. 2013;8(1):e54822.

14. Nicoletti A, Luca A, Luca M, et al. Obsessive-compulsive personality disorder in drug-naive Parkinson's disease patients. J Neurol. 2015;262(2):485-486.

15. Harbishettar V, Pal PK, Reddy YCJ, et al. Is there a relationship between Parkinson's disease and obsessive-compulsive disorder? Parkinsonism and Related Disorders. 2005;11(2):85-88.

16. Maia AF, Pinto AS, Barbosa ER, et al. Obsessive-compulsive symptoms, obsessive-compulsive disorder and related disorders in Parkinson's disease. J Neuropsychiatry Clin Neurosci. 2003;15(3):371-374.

17. Joel $\mathrm{D}$, Zohar $\mathrm{O}$, Afek $\mathrm{M}$, et al. Impaired procedural learning in obsessive-compulsive disorder and Parkinson's disease, but not in major depressive disorder. Behav Brain Res. 2005;157(2):253-263.

18. Merola A, Romagnolo A, Rizzi L, et al. Impulse control behaviors and subthalamic deep brain stimulation in Parkinson disease. $J$ Neurol. 2017;264:40-48.

19. Cheyette SR, Cummings JL. Encephalitis lethargica: lessons for contemporary neuropsychiatry. $J$ Neuropsychiatry Clin Neurosci. 1995;7(2):125-134

20. Robinson D, Wu H, Munne RA, et al. Reduced caudate nucleus volume in obsessive-compulsive disorder. Arch Gen Psychiatry. 1995;52(5):393-398.

21. Evans AH, Katzenschlager R, Paviour D, et al. Punding in Parkinson's disease: its relation to the dopamine dysregulation syndrome. Movement Disorders. 2004;19(4):397-405.

22. Nicoletti A, Luca A, Raciti L, et al. Obsessive Compulsive Personality Disorder and Parkinson's Disease. PLoS ONE. 2013;8(1):e54822.

23. Baxter LR, Phelps ME, Mazziotta JC, et al. Local cerebral glucose metabolic rates in obsessive-compulsive disorder: a comparison with rates in unipolar depression and in normal controls. Arch Gen Psychiatry. 1987;44(3):211-18.

24. Laplane D, Lavasseur M, Pillon B, et al. Obsessive-compulsive and other behavioural changes with bilateral basal ganglia lesions. A neuropsychological magnetic resonance imaging and positron tomography study. Brain. 1989;112(3):699-725.

25. Mallet L, Mesnage V, Houeto JL, et al. Compulsions, Parkinson's disease and stimulation. Lancet. 2002;360(9342):1302-1304.

26. Alexander GE, Delong MR, Strick PL. Parallel organization of functionally segregated circuits linking basal ganglia and cortex. Annu Rev Neurosci. 1986;9:357-381.

27. Alexander GE, Crutcher MD, Delong MR. Basal ganglia-thalamocortical circuits: parallel substrates for motor, oculomotor, prefrontal and limbic functions. Prog Brain Res. 1990;85:119-46.
28. Holthoff-Detto VA, Kessler J, Herholz K, et al. Functional effects of striatal dysfunction in Parkinson disease. Arch Neurol. 1997;54(2):145150

29. Eidelberg D, Moeller JR, Ishikawa T, et al. Assesment of disease severity in parkinsonism with fluorine -18- fludrodeoxyglucose and PET. J Nucl Med. 1995;36(3):378-383.

30. Markus HS, Costa DC, Lees AJ. HMPAO SPECT in Parkinson's disease before and after levodopa: correlation with dopaminergic responsiveness. J Neurol Neurosurg Psychiatry. 1994;57(2):180-185.

31. Williams NR, Hopkins TR, Short EB, et al. Reward circuit DBS Improves Parkinson's Gait along with severe depression and OCD. Neurocase. 2016;22(2):201-204.

32. Greer JM, Capecchi MR. Hoxb8 is required for normal grooming behavior in mice. Neuron. 2002;33(1):23-34

33. Nordstrom EJ, Burton FH. A transgenic model of comorbid Tourette's syndrome and obsessive-compulsive disorder circuitry. Mol Psychiatry. 2002;7(6):617-625, 524

34. Welch JM, Lu J, Rodriguiz RM, et al. Cortico-striatal synaptic defects and OCD-like behaviours in Sapap3-mutant mice. Nature. 2007;448(7156):894-900.

35. Shmelkov SV, Hormigo A, Jing D, et al. Slitrk5 deficiency impairs corticostriatal circuitry and leads to obsessive-compulsive-like behaviors in mice. Nat Med. 2010;16(5):598-602.

36. Ting JT, Feng G. Neurobiology of obsessive-compulsive disorder: insights into neural circuitry dysfunction through Mouse genetics. Curr Opin Neurobiol. 2011;21(6):842-848.

37. Cummings JL. Frontal-subcortical circuits and human behavior. Arch Neurol. 1993;50(8):873-880.

38. Cummings JL, Frankel M. Gilles de la Tourette syndrome and the neurological basis of obsessions and compulsions. Biol Psychiatry. 1985;20(10):1117-1126.

39. Junque C, Alegret M, Nobbe FA, et al. Cognitive and behavioral changes after unilateral posteroventral pallidotomy: relationship with lesional data from MRI. Mov Disord. 1999;14(5):780-789.

40. Saint-Cyr JA, Taylor AE, Nicholson K, et al. Behavior and the basal ganglia. Adv Neurol. 1995;65:1-28.

41. Austin MP, Mitchell P. The anatomy of melancholia: does frontalsubcortical pathophysiology underpin its psychomotor and cognitive manifestations? Psychol Med. 1995;25(4):665-672.

42. Daniele A, Bartolomeo P, Cassetta E, et al. Obsessive-compulsive behaviour and cognitive impairment in a parkinsonian patient after left putaminal lesion. J Neurol Neurosurg Psychiatry. 1997;62(3):288-289.

43. Rauch SL, Dougherty DD, Shin LM, et al. Neural correlates of factoranalyzed OCD symptom dimensions: a PET study. CNS Spectr. 1998;3:37-43. 